\title{
Conclusive analysis and cause of the flyby anomaly
}

\author{
V. Guruprasad \\ Inspired Research, New York, USA
}

\begin{abstract}
JPL's own data correlate to 1\% NEAR and Rosetta trajectory discrepancies to an unexpected doubling of path times in phase locked tracking. NEAR's radar residuals illustrate the doubling to $\mathbf{5} \sigma$. Analysis of these and other NASA-tracked flybys shows that a distance sensitive anomalous signal generally exists.
\end{abstract}

\section{INTRODUCTION}

Deep space tracking exploits the carrier Doppler shift and the round trip times of range codes modulated in the telemetry signal to continuously track the (radial) velocity and range of spacecraft. The Doppler has the advantages of insensitivity to ground clock timing jitter and independence from modulation, and can be measured to well below $1 \mathrm{~mm} \mathrm{~s}^{-1}$; the range codes complement Doppler-integrated range [1, §III].

As used by JPL authors, the term "flyby anomaly" refers to apparent velocity discrepancies between pre- and post-perigee telemetry tracks in Earth flybys used for gravitational assist, as noticed notably in the flybys of Galileo (1990), NEAR (1998) and Rosetta (2005) [2]. Their interpretation as actual velocity changes, which could entail changes to the laws of gravitation, is problematic, however, without independent corroboration to eliminate a telemetry systematic, given that a similar anomaly was eventually traced to an overlooked systematic [3]-[5].

Coherent radars of the Space Surveillance Network (SSN) indeed placed NEAR consistently closer by up to $1 \mathrm{~km}$ while in geostationary range. This exceeds their $5-25 \mathrm{~m}$ precisions [6], [7] by two orders [8]. Radar is susceptible to clock jitter, like the range codes, but it bypasses the transponder, and thus is an independent test of the telemetry tracking. This pattern of radar residuals, extrapolated via a back-of-envelope trajectory estimate to the post-perigee re-acquisition of signal (AOS) by Deep Space Network (DSN) station DSS Canberra, explained $80 \%$ of the $13.46 \mathrm{~mm} \mathrm{~s}^{-1}$ anomaly [9]. With more precise range and range rate from JPL Horizons [10] using the official trajectory [11], the corresponding error at loss of signal (LOS) by DSS Goldstone increases the total error to $13.64 \mathrm{~mm} \mathrm{~s}^{-1}$, only $1.34 \%$ off the anomaly value obtained by JPL.

The pattern revealed by JPL's original scatter plot in Fig. 1 is an apparent excess travel time $\Delta t$ for telemetry signals that fits one-way light time $r / c$, as shown by the overlaid plots of range $r$ (dashed) from the radars and range lags $\Delta r=-v r / c$. The data fit the lags within respective precisions (dotted lines) but for Millstone's initial points at its limit of range. The radars tracked after LOS [8], so JPL's trajectory used as reference for the residuals was extrapolated using the last Doppler at LOS. The negative residuals thus signify a systematic Doppler offset proportional to instantaneous range in the telemetry.

Any notion of distance dependence in frequency is of course deeply disturbing. It questions, for example, the conservation

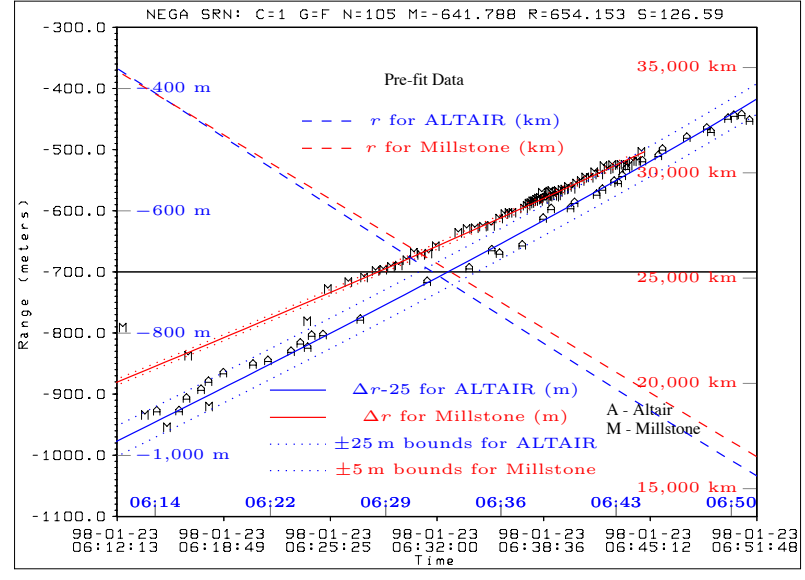

Fig. 1: NEAR's radar residuals and one-way range lags

of photon energies. Inference of the cosmological expansion from the Hubble shifts also requires its impossibility.

However, the only possible alternative ${ }^{1}$ explanation for the range errors in Fig. 1 is actual difference in speed [12] between telemetry and radar over virtually the same paths, in violation of relativity. The residuals as such also defy exotic hypotheses like dark matter [14], [15] and systematics including the solar wind, spacecraft charging, magnetic moments, tides, etc. [2], that affect both paths. The only significant physical difference between the two paths is the onboard transponder.

The velocity anomaly has not reappeared in NASA missions since Cassini [16], including MESSENGER ${ }^{2}$, EPOXI [18] and Juno [19]; this was privately posed [17] as a challenge to [9]. A correlation with newer transponder technology called SDST was then noticed, in which the loop error is computed digitally i.e., at intervals of the (second) intermediate frequency (IF) [20], and could block the anomalous chirp modes proposed in [9]. The phase comparison was performed at (the second) IF also on NEAR [21], so the issue is whether the local oscillator (LO) followed the RF and IF carriers continuously, or in steps.

Cassini had a transponder of the same design as NEAR [22],

\footnotetext{
${ }^{1}$ We have two velocities, of light $c$ or of the spacecraft $v$, for relating the residuals $\Delta r$ to time. Using $v$ leads to $\Delta t=-\Delta r / v$ and to lags in velocity $\Delta v=-a r / c$ and Doppler $\Delta \omega=-\omega \beta r / c$ for Doppler rate $\beta \approx a / v$ [9], to explain the anomaly. The Lorentz correction is about $10^{-10}$ in the flybys, so non-relativistic formulas are adequate. The alternative $\Delta t=-\Delta r / c$ is not only aether-theoretic [13], but retains the range proportionality that motivated its proposal [12] in the first place.

${ }^{2}$ According to Campbell [17], "The group of authors of our 2008 PRL paper fit an orbit to $+/-4$ days of Doppler and had no need to introduce an energy correction to obtain a satisfactory result."
} 
but with no gaps in the tracking of its flyby, a velocity jump could not have stood out. The interleaving of six tracks [16, Fig. 13a] would have averaged out station-specific shifts like this range specific Doppler error, and its swings indeed go only to $\pm 0.15 \mathrm{~mm} \mathrm{~s}^{-1} \equiv \pm 2 \mathrm{mHz}$. The average of excess travel times cannot cancel out, however. Cassini's periapse advanced relative to the target set at the last trajectory control manoeuvre (TCM) by $1 \mathrm{~s}$. A similar $0.34 \mathrm{~s}$ advance in Rosetta's 2005 flyby fits the JPL Horizons $331.2 \mathrm{~ms}$ two-way light time to $2.6 \%$.

The closest approach of OSIRIS-REx was $17,237 \mathrm{~km}$, and the closest EPOXI flyby was at $15,567 \mathrm{~km}$, so their residuals are not meaningful for comparing. MESSENGER came closer at $2,348 \mathrm{~km}$, almost as close as Rosetta in 2005 . Its X-band residuals remain below $\pm 4 \mathrm{mHz} \sim \pm 0.3 \mathrm{~mm} \mathrm{~s}^{-1}$, and display no offset across the gap in tracking at the periapse. Juno came almost as close as NEAR. Its tracks had multi-path artefacts, and the residuals remained $\pm 1 \mathrm{~mm} \mathrm{~s}^{-1}$, but only due to spin [19], [23]. We thus have a paradox that tracking the RF carrier continuously in Earth flybys causes large unexpected Doppler residuals and often also velocity errors, but catching up only at IF intervals achieves perfect agreement with standard physics! Current technology is somehow specific to this approximation and inconsistent with some circumstance in the flybys.

This approximation is revealed by the uplink section of the New Horizons transponder schematic from [24] in Fig. 2, in which the digitally controlled oscillator (DCO) of EPOXI and MESSENGER SDSTs was replaced by direct digital synthesis (DDS) [25]. As the DDS is corrected by digital samples via the digital filter $F(s)$ at IF intervals, the DDS, and thus the LO inputs to the down-conversion mixers are steady over multiples of RF/IF $\approx 2873 \mathrm{RF}$ cycles. The WBIF and demodulated range outputs are from the product of a changing first stage IF carrier with a sinusoid for $240 / 2.5=96$ cycle intervals. The digital design requires that the total phase variation over an IF period be below a cycle. During the SSN tracking, the non-relativistic, fractional Doppler rate $\beta=a / v$ was around $10^{-5} \mathrm{~s}^{-1}$, so the total Doppler change was $29 \mathrm{mHz}$ over the $2.5 \mathrm{MHz} \sim 0.4 \mu \mathrm{s}$ IF period. The SDSTs could not have had "cycle slips".

The analogue phase locked tracking had more information, however, in the form of range dependence in the Doppler error. Current notions against its possibility are why some untenable hypotheses arose and the SSN residuals, also seen for Galileo [17], were disregarded as "noise". This was despite JPL having standard deviation $S=126.59 \mathrm{~m}$ and root mean square error $R=654.153 \mathrm{~m}$ as annotated in Fig. 1, which already qualified them as $5.1675 \sigma$ [11]. Ignoring the initial out-of-range points reduces $R$ slightly. Using its $5 \mathrm{~m}$ precision as $\sigma$, Millstone's points exceed $100 \sigma$, and were limited only by range.

The only circumstance in the flybys we can relate to such a change in transponders is Doppler rates in hyperbolic approach and retreat, with accelerations below $1 \mathrm{~m} / \mathrm{s}^{2}$. We experience greater accelerations in elevators, for example, but too briefly for similar observations. The telemetry based tracking is meant for deep space and not used for satellites.

We find a precedent for range proportional shifts in FMCW radars, which use a continuous signal with frequency ramped over time as $\omega(t)=\omega_{0}[1+\gamma t]$. The lag $\Delta \omega=-\omega_{0} \gamma r / c$ over a round trip of length $r$ is typically determined using an FFT, as a measure of $r$. At a steady acceleration $a \equiv \ddot{r}$, the Doppler rate $\beta=a / v$ would lead to an exponential $\operatorname{ramp} \omega(t)=\omega_{0} e^{\beta t}$ but this is identical to the first order with $\gamma=\beta$. The received frequency is exactly what was emitted by the source, however, so no correction has been needed in trajectory software, called orbit determination (OD), for this "FMCW shift".

The Doppler rate lags $\Delta \omega=-\omega \beta r / c$ in Fig. 1 thus amount to an unmodelled doubling of the FMCW lag $\Delta \omega$. No doubling occurs, consistent with FMCW, only with the SDSTs, which ensure a steady LO in down-converting each IF frame. The anomaly itself or some of its symptoms are seen in all missions in which the LO phase locked to Doppler, viz Galileo, NEAR, Rosetta and likely Cassini, as stated. All phase locked Doppler rate observations thus contradict current notions.

A core principle of radar and communication, that all wave transport is inherently distance insensitive, is thus contradicted. The magnitudes of the SSN residuals, their clear linearity, and the independence of radar from onboard processing refute their rejection as noise, and their only alternative explanation would violate constancy of speed of light, at a range populated by GPS and other satellites. The prevailing ideas of frequencies varying in time but unchanged by travel, seem to hold only if the receiver LO does not continuously ramp.

Naïvely, one might write $\psi_{L}=\exp \left(i \omega_{0}[1+\gamma t][t-r / c]\right)$ for the FMCW echo, to describe location-independent variation of frequency, but $\psi_{L}$ does not even satisfy the wave equation. As $t$ occurs in more terms within the phase than $r$, its second order derivatives $\partial^{2} \psi_{L} / \partial t^{2}$ and $c^{2} \partial^{2} \psi_{L} / \partial r^{2}$ can at all be equal only at the origin (source). The issue does not arise if $\omega$ is constant, which is true for each of its Fourier components, but a constant $\omega$ cannot denote a changing Doppler. A Doppler rate $\beta$ means component frequencies also change as $\omega(t)=\omega_{0} e^{\beta t}$, yielding the phase $\phi(t)=\int \omega(t) d t=\omega_{0} e^{\beta t} / \beta$.

We thus get $\psi_{E}=\exp \left(i \omega_{0} e^{\beta[t-r / c]} / \beta\right)$ including the travel delay $r / c$. It is symmetric in $t$ and $r$, and also satisfies the wave equation, but has shifts $\omega_{0} e^{-\beta r / c}$ for displacements $r / c$. Shifts also occur in $\psi_{L}$ if we changed $\gamma t$ to $\gamma[t-r / c]$ to satisfy the wave equation and refer frequencies to source time. But they seem to add over the "FMCW shifts" only with phase lock.

Another issue is that any uniform shifts $\omega \rightarrow \omega[1+z]$ signify a time dilation via Fourier inversion as $\int F(\omega[1+z]) e^{i \omega t} d t=$ $(1+z)^{-1} f(t /[1+z])$, so the older transponders seem to have received physically different waves altogether!

The next section presents the correlation of NEAR's velocity anomaly and post-encounter Doppler oscillations with velocity lags as inferred above from the SSN radar residuals of Fig. 1. Section III discusses similar correlations for Galileo 1990 and Rosetta 2005 flybys, which too exhibited velocity anomalies. Section IV shows that this pattern is consistent with the known absence of the velocity anomaly in both of Rosetta's 2007 and 2009 flybys if we take into account the carrier loop bandwidth, and treats the perigee shifts in more detail.

These correlations, found even without velocity anomalies, lead to the conclusion that an "anomalous" signal with distance 


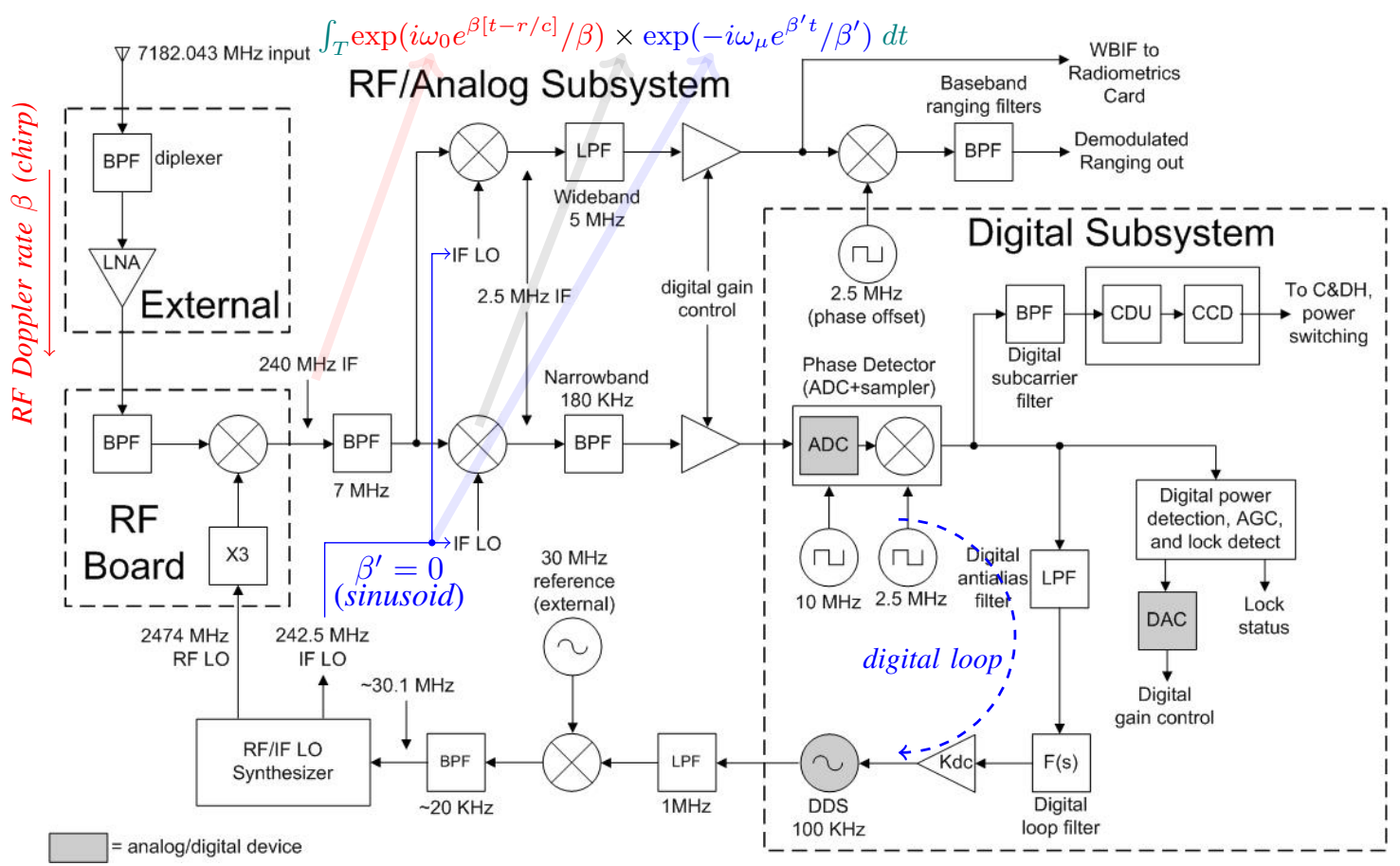

Fig. 2: Carrier loop and Hilbert product in the New Horizons transceiver [24]

sensitivity like $\psi_{E}$ is always present but observable only with a similarly varying $\mathrm{LO}$, as ensured by the continuous phase lock in older transponders. The distance-invariant "normal" signal of current theory, received under coarser digital tracking at IF, seems to require $\psi_{L}$ inconsistent with the wave equation. How both these signals might be observable from the same physical waves is explained in Section V. The validity of $\psi_{E}$ as a wave function is proved in the appendix for completeness.

\section{CORRELATION With VELOCITY LAGS}

Fig. 3 shows the range and range rate given by JPL Horizons for Goldstone (DSS 25) over a $12 \mathrm{~h}$ window around the perigee in NEAR's 1998 flyby, and the velocity lag given by the range rate $v$ and radial acceleration $a=d v / d t$ as $\Delta v=-a r / c$. The velocity lag should result in the form of the Doppler lag $\Delta \omega$ if the latter were the cause of the anomalous velocity gain.

The graph shows that the lag had a maximum of $7 \mathrm{~mm} \mathrm{~s}^{-1}$, but was only $2.51 \mathrm{~mm} \mathrm{~s}^{-1}$ at LOS. The corresponding plots for Canberra (DSS 34) (Fig. 4) show a close to maximum lag of $11.10 \mathrm{~mm} \mathrm{~s}^{-1}$ at AOS. The lags signify that the telemetry Doppler had a too small a value at LOS and a larger one than the actual speed at AOS, so the sum of these errors should fit JPL's value for the anomalous velocity gain. The estimate of 10.7 in [9] is thus corrected to $11.13+2.51=13.64 \mathrm{~mm} \mathrm{~s}^{-1}$, $1.3 \%$ of JPL's $13.46 \mathrm{~mm} \mathrm{~s}^{-1}$ [2], for the velocity discrepancy across the $2 \mathrm{~h} 39$ min gap in tracking at perigee.

The lags must also distort the trajectory. OD only minimizes mean square error, and cannot detect smooth distortions in the trajectory because that would require precise knowledge of all external forces. As a result, velocity mismatches across gaps in tracking as in Galileo's and NEAR's flybys were the only way to definitively detect such tracking anomalies. Remarkably, the Doppler residuals do reveal actual distortions, as follows.

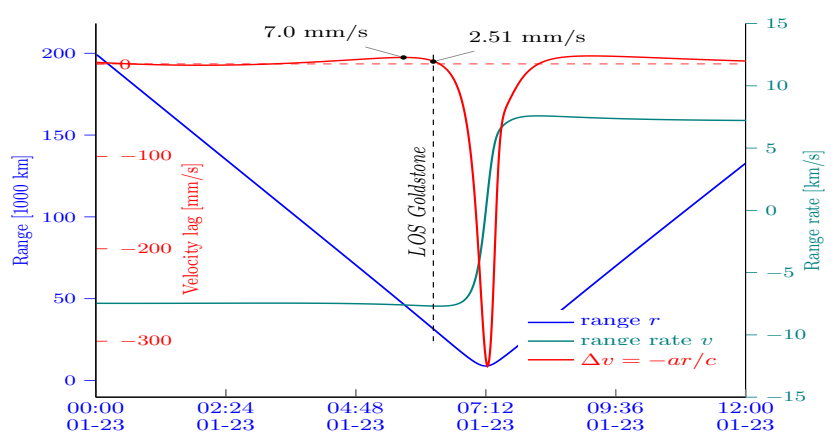

Fig. 3: Analysis for NEAR's Goldstone track

The range, range rate and velocity lag are shown in Fig. 4 overlaid on the post-perigee Doppler residual oscillation from Fig. 4 of [8]. The Doppler follows the lag and is a quarter cycle behind the range rate, so the OD's notion of the instantaneous velocity was dragged by the Doppler lag, like a force function driving damped oscillations in a spring. This phase difference between the Doppler and range rate had led to a suspicion of a direction prediction problem in OD [2].

The lag increases with range $r$ as the deceleration $a$ due to gravity is changing slowly. The steady amplitude seen in the residuals must then mean a growing oscillation within the JPL Horizons trajectory, but the range rate oscillations in flybys are caused by Earth's rotation [1, §III-B.3], and cannot grow. 
The last pair of peaks in the range rate from JPL Horizons are 0.2255 and $0.2278 \mathrm{~km} \mathrm{~s}^{-1}$ above their preceding troughs, meaning a growth of $2.3 \mathrm{~m} / \mathrm{s}$ per day. This is a feature present in JPL's inferred trajectory that is exposed by our analysis. It also obviates a closer fit to the velocity anomaly.

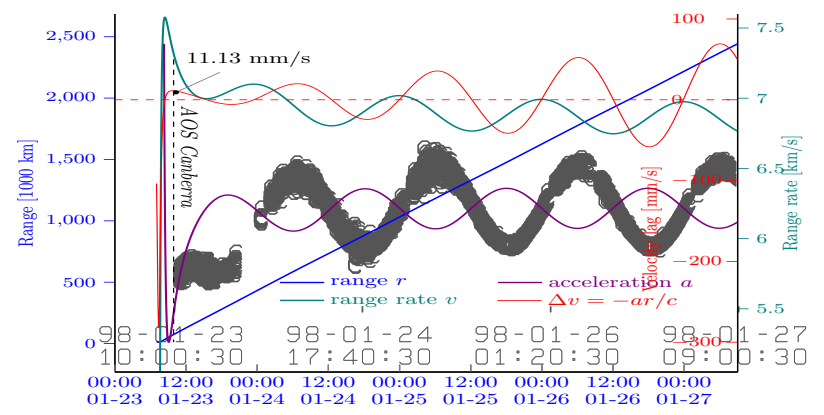

Fig. 4: Correlation with NEAR's Canberra track

\section{RESIDUALS IN OTHER FLYBYS}

This close fit to the NEAR velocity anomaly was fortuitous. The lags were changing slowly at LOS and AOS, hence a few minutes of error in LOS and AOS times did not matter.

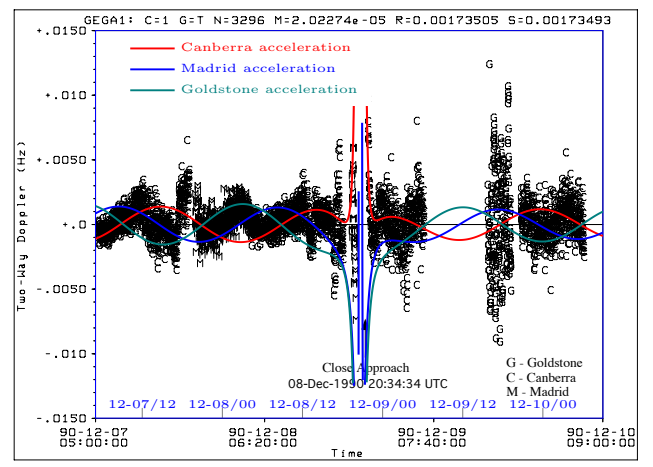

Fig. 5: Galileo 1990 accelerations over Doppler [8, Fig. 3]

In flybys closely tracked near the perigee, the lags would be changing rapidly at AOS and LOS times, so small uncertainties in these times should make a close fit to OD-computed velocity mismatch unlikely. Extreme data points at the start and at the end could be also discarded in OD as outliers. The computed anomaly cannot be exceed the sum of the LOS and AOS lags, however. Also, the Doppler residuals should also follow the radial acceleration instead of range rate, as in Fig. 4 for NEAR.

Fig. 5 shows the differenced range rates for all three tracking stations in Galileo's 1990 flyby, overlaid on Doppler residuals where the anomaly was removed by estimation (Fig. 3 of [8]). Goldstone's post-perigee track had to be discarded [8], so the Canberra track was alone responsible for the anomaly and does follow the acceleration around the perigee.

Fig. 6 shows the computed velocity lags were $26 \mathrm{~mm} \mathrm{~s}^{-1} \approx$ $0.4 \mathrm{~Hz}$ at LOS Madrid, and $-57 \mathrm{~mm} \mathrm{~s}^{-1} \approx-0.87 \mathrm{~Hz}$ at AOS Canberra. Both are within the nominal carrier loop bandwidth of $1 \mathrm{~Hz}$, but far off the $4.3 \mathrm{~mm} \mathrm{~s}^{-1}$ anomaly value.
In Rosetta's 2005 flyby, a $1.82 \mathrm{~mm} \mathrm{~s}^{-1}$ impulse annuls the $3.6 \mathrm{~mm} \mathrm{~s}^{-1}$ offset of the post-perigee residuals [26]. We need to use two-way lags and offset for Rosetta as the uplink is not ramped in ESA missions [27]. The lags for New Norcia, which tracked closest to perigee, are 10.15 at LOS before perigee and $206.8 \mathrm{~mm} / \mathrm{s}$ at the following AOS. The latter signifies a shift of $5.8 \mathrm{~Hz}$, so the anomalous signal would be filtered away.

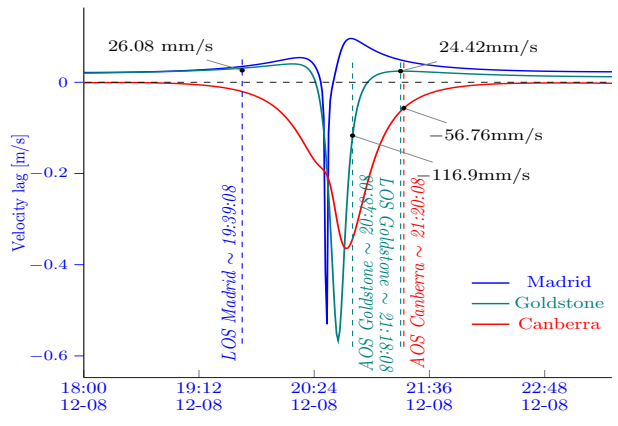

Fig. 6: Galileo 1990 velocity lags

Only the unshifted normal signal was thereby available for lock in the post-perigee New Norcia track. The LOS lag value would allow residuals up to $10.15-3.6=6.55 \mathrm{~mm} / \mathrm{s}$ but they only reach $1.8 \mathrm{~mm} / \mathrm{s}$, as the spacecraft was not continuously in view. The residual clusters seen are individually reacquired two way tracks, unlike the essentially continuous post-perigee track for NEAR in Fig. 4. The trajectory was computed as a best fit across all nine days, so no velocity mismatch is seen in Fig. 5 of [26], revisited below in Figs. 7 and 8 . The $3.6 \mathrm{~mm} / \mathrm{s}$ offset was revealed separately in their Fig. 6 by zero-weighting the post-perigee data [26]. We would need the start and end times for each day's tracks and compute a corresponding mean value of the velocity lags to attempt a quantitative fit. We do have correlation with the acceleration $a$, however, as revealed in Fig. 7 by overlaying the range rate and acceleration given by JPL Horizons for New Norcia on Fig. 5 of [26] at a vertical scale adjusted to expose the correlation visually.

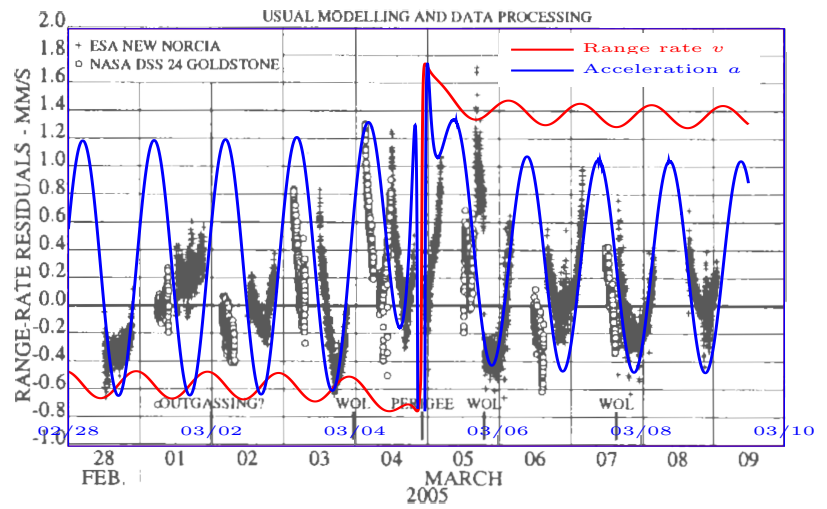

Fig. 7: Correlation with Rosetta 2005 New Norcia track

Even the mismatch of a few hours on 03 and 04 March can be removed by adjusting the vertical scale of the acceleration, without changing its diurnal phase. The post-perigee swings 
from -0.2 to $1.1 \mathrm{~mm} / \mathrm{s}$, and from 1.7 back to $0.5 \mathrm{~mm} / \mathrm{s}$, on 05 March again coincide with cusps in the acceleration.

Fig. 8 shows the overlaid range rate and acceleration from JPL Horizons for Goldstone. The only points out of step with the radial acceleration are the last track from Goldsone on 07 March, consistent with the New Norcia data being used for the trajectory. The $-0.6 \mathrm{~mm} / \mathrm{s}$ outlier on 01 March also seems to no longer require outgassing as explanation.

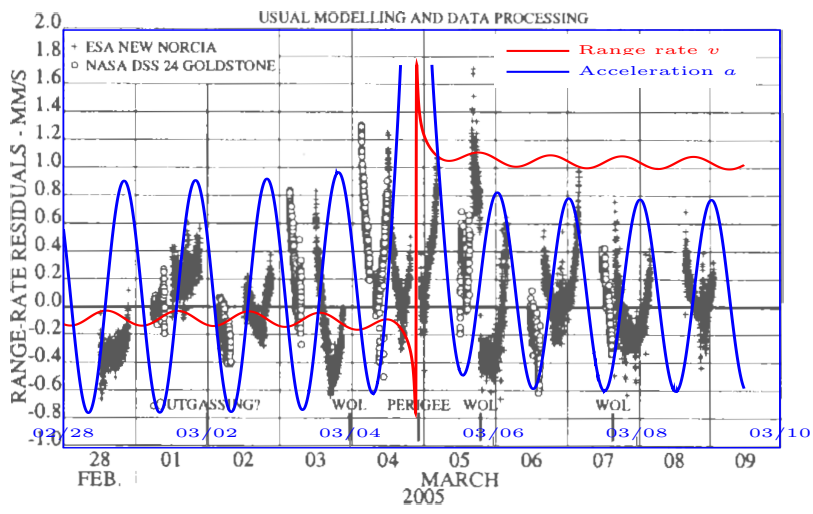

Fig. 8: Correlation with Rosetta 2005 Goldstone track

\section{ABSENCES AND PERIGEE SHIFTS}

The known absences of the anomaly in other flybys with the RF phase lock transponders are consistent with the anomalous signal being blocked by the RF tuning if not the loop filter. For Rosetta's 2007 flyby, JPL Horizons data yields the velocity lag of $514.6 \mathrm{~mm} / \mathrm{s}$ for Goldstone AOS, which far exceeds the usual $1 \mathrm{~Hz}$ carrier loop bandwidth, so only the normal signal was admitted and followed. The $41.76 \mathrm{~mm} / \mathrm{s} \equiv 1.18 \mathrm{~Hz}$ New Norcia LOS lag is also large, but the normal signal was likely already acquired, precluding the anomaly.

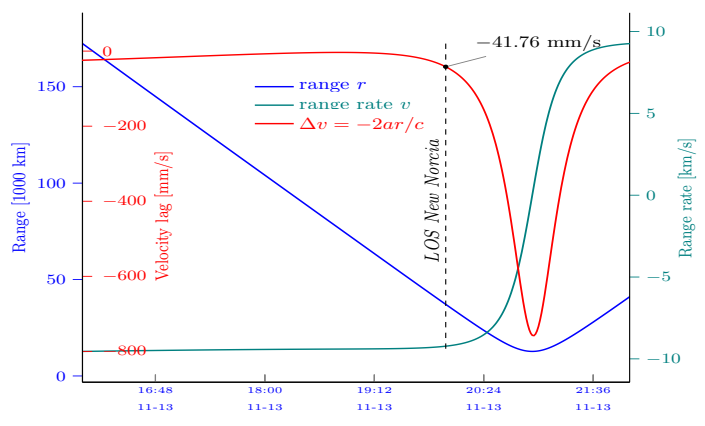

Fig. 9: Analysis for Rosetta 2007 New Norcia track

In 2009, the initial tracking from Kourou yields velocity lags of 0.3 and $0.5 \mathrm{~Hz}$ at start and end, respectively, but the track ended long before the perigee. In the post-perigee track from Maspalomas, the lag was initially $-600 \mathrm{~mm} / \mathrm{s}$, too large for acquiring the anomalous signal. As the lock remains till end of track, the smaller $-4.4 \mathrm{~mm} / \mathrm{s}$ lag at the end is inconsequential.

New Norcia actually tracked through perigee, starting with a lag of only $0.04 \mathrm{~mm} / \mathrm{s} \equiv 1.11 \mathrm{mHz}$ so a half-cycle of phase difference would have taken $450 \mathrm{~s} \equiv 7.5 \mathrm{~min}$. The lag then increases, and is $-530 \mathrm{~mm} / \mathrm{s}$ at the end of New Norcia track. As this is a lag, the Doppler changes first in the normal signal, so the latter controlled the phase error and lock, again preventing the anomaly.

The lags should have also displaced the perigee, as the Earth would have moved $10.48 \mathrm{~km}$ in its orbit in the one-way light times of $103.67 \mathrm{~ms}$ at LOS and $245.61 \mathrm{~ms}$ at AOS in NEAR's flyby, for instance. There is a $6.8 \mathrm{~km}$ difference between the altitude of $532 \mathrm{~km}$ intended after the last TCM (post-Mathilde burn) [28] and $538.83 \mathrm{~km}$ reported after the flyby [8].

A similar displacement of $1.4 \mathrm{~mm} / \mathrm{s} \times 167 \mathrm{~min} \approx 14.03 \mathrm{~km}$ was reported in Rosetta's 2005 flyby, with a perigee advance of $0.34 \mathrm{~s}$, which fits the two-way light time to $2.6 \%$, as remarked.

These deviations were accepted as navigational tolerance, in absence of independent validation of TCMs and the ephemeris at the last TCM range and precision. The close fit of Rosetta's advance, and the drop in perigee shift by a full order to $26 \mathrm{~ms}$ with opposite sign in Juno's flyby [19], [23], suggests that the anomaly had been limiting navigational tolerance.

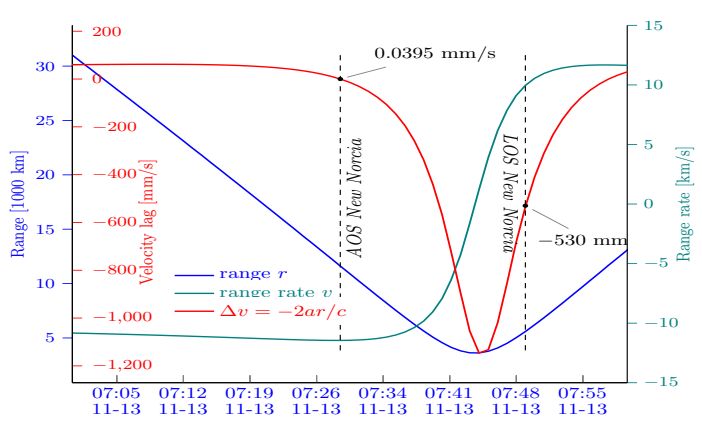

Fig. 10: Analysis for Rosetta 2009 New Norcia track

\section{ROLE OF THE RECEIVER DESIGN}

Figs. 11a and 11b illustrate the observed spectra of a signal with a Doppler rate propagating according to current ideas, and as revealed by radar residuals in Fig. 1, respectively, between the source and receiver frequency axes. The wavelengths are shown for four instants by colours assuming a positive Doppler rate. Also shown are the basis vectors $\mathbf{e}_{\mu}=e^{i \omega_{\mu} t}$ in the Hilbert space formalism of the Fourier spectrum, having coefficients $F_{\mu}=\mathbf{e}_{\mu}^{*} \cdot \psi_{E} \equiv T^{-1} \int_{T} e^{-i \omega_{\mu} t} e^{i \omega_{0} e^{\beta[t-r / c]} / \beta} d t$ for $\psi_{E}$.

A basis vector $\mathbf{e}_{\mu} \sim e^{-i \omega_{\mu} t}$ is generated by the IF LO, and multiplied with the incoming signal $\psi_{E}$ at the second stage IF mixers, as highlighted in the receiver schematic, Fig. 2. As is well known, the multiplication yields both sum and difference frequencies, and the IF filters suppress the sums. The IF LO is negative in the difference terms, so the mixing and filtering in effect also perform conjugation of $\mathbf{e}_{\mu}$ to $\mathbf{e}_{\mu}^{*}$.

The digital filter $F(s)$ is what limits the loop bandwidth to $\sim 1 \mathrm{~Hz}$, so its time constant is effectively $1 \mathrm{~s}$, but it updates the DDS at each IF sample, at $2.5 \mathrm{MHz} \sim 0.4 \mu$ s intervals. This IF interval is also a safe estimate for the integration time $T$ effectively applied when the IF output is consumed. 
Fig. $11 \mathrm{~b}$ shows the basis vectors $\mathbf{e}_{\mu}^{\prime}$ as in effect implemented in the older transponders. They are inclined upward to the right of the receiver frequency axis since their frequencies increase with $t$ for $\beta^{\prime}>0$. As the phase locked IF LO followed the carrier's each cycle, it shared the latter's phase acceleration, so its instantaneous basis vector is $\mathbf{e}_{\mu}^{\prime}=\exp \left(i \omega_{\mu} e^{\beta^{\prime} t} / \beta^{\prime}\right)$ where $\beta^{\prime}=\beta$, the fractional Doppler rate $\dot{\omega} / \omega$ of the IF carrier. The Hilbert products with the Doppler rate signal, $\mathbf{e}_{\mu}^{\prime *} \cdot \psi_{E}$, are

$$
\begin{aligned}
F_{\mu}^{\prime} & =\frac{1}{T} \int_{T} \exp \left(-i \omega_{\mu} e^{\beta^{\prime} t} / \beta^{\prime}\right) \exp \left(i \omega_{0} e^{\beta[t-r / c]} / \beta\right) d t \\
& \simeq \delta\left(\beta^{\prime}-\beta\right) \delta\left(\omega_{\mu}-\omega_{0} e^{-\beta r / c}\right) \quad \text { for } T \gg 2 \pi / \omega_{\mu} .
\end{aligned}
$$

Phase lock means both delta functions are unity, at which the integrand is unity and the amplitudes $F_{\mu}^{\prime}$ have offsets $\Delta \omega_{\mu} \approx$ $-\omega_{\mu} \beta r / c$. We thus have $\left|F_{\mu}^{\prime}\right| \sim 1$, even in the limit $T \rightarrow \infty$.

The result reduces to Fourier at $\beta=0$, so $F_{\mu}^{\prime}$ are the Fourier coefficients displaced by $\Delta \omega_{\mu}$ if $\beta \neq 0$. The chirp spectrum is the Fourier transform at non-zero Doppler rates. It is indeed the spectrum received under continuous phase lock [9].

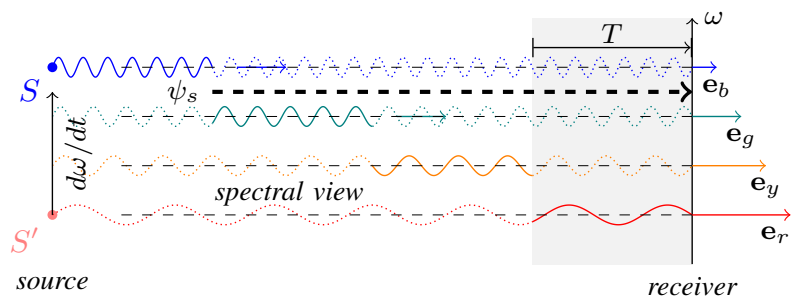

(a) Propagation in the Fourier basis

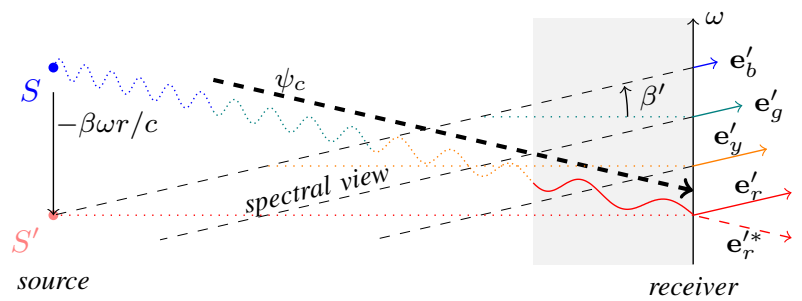

(b) Chirp basis and inclined spectral view

Fig. 11: Waves and their spectra

In contrast, each Fourier coefficient $F_{\mu}$ is contributed by a limited set of cycles in $T$ across which the basis frequency $\omega_{\mu}$ and the signal $\exp \left(i \omega_{\mu} e^{\beta[t-r / c]} / \beta\right)$ differ in phase by less than a cycle. This can be quantified with the total change parameter $\Delta=e^{\beta T}-1 \approx \beta T \approx 4 \times 10^{-12}$ for the SDST of Fig. 2. It yields $\Delta \omega_{c} \equiv \Delta \times \omega_{c}=0.029 \mathrm{~Hz}$ as the total Doppler change over $T$, and $T \Delta \omega_{c} \approx 1.15 \times 10^{-8}$ cycles for the total phase difference, so every cycle contributes to $F_{\mu}$.

It is possible to have $\Delta$ so large that $\Delta \omega_{c}>T^{-1}$, in which case, only a few cycles can contribute, so $\left|F_{\mu}\right| \ll\left|F_{\mu}^{\prime}\right|$, since everywhere else in $T$, the integrand becomes a beat oscillation with net zero contribution. One way this can happen is if $\beta>$ $1 / \omega_{c} T^{2} \approx 870$ for the SDST of Fig. 2 . This would be a very large fractional acceleration, and could occur if $v$ were initially close to zero. The other possibility is $T>1 / \sqrt{\omega_{c} \beta} \approx 3.7 \mathrm{~ms}$, which could, for instance, affect OFDM. It is safe to conclude that $\left|F_{\mu}\right| \rightarrow 0$ as $T \rightarrow \infty$, meaning Doppler rate signals are really not represented by the Fourier transform. A closed form for this indefinite integral is as yet unavailable.

Although $\left|F_{\mu}\right|$ would be slightly smaller than $\left|F_{\mu}^{\prime}\right|$ and differ a little in phase, their difference is too small to explain the specificity of the shifts to phase lock. The shifts are due to the inclination of the components $F_{\mu}^{\prime} \mathbf{e}_{\mu}^{\prime} \sim F_{\mu}^{\prime} \exp \left(i \omega_{\mu} e^{\beta^{\prime} t} / \beta^{\prime}\right)$ alone, as any reference to an earlier time $(t-\tau)$ means going along the inclined dashed lines as $F_{\mu}^{\prime} \exp \left(i \omega_{\mu} e^{\beta^{\prime}[t-\tau]} / \beta^{\prime}\right)$, and reaching the source at $\tau=r / c$. The coefficients $F_{\mu}^{\prime}$ conversely must fit the source when extrapolated this inclined way. This is precisely what the Hilbert product $\mathbf{e}_{\mu}^{\prime *} \cdot \psi_{E}$ computes.

The propagation of $F_{\mu}^{\prime} \mathbf{e}_{\mu}^{\prime}$ is thus inclined, as indicated by the thick dashed line $\psi_{c}$, so the components expand en route.

The Fourier components of Fig. 11a are defined by $F_{\mu} \mathbf{e}_{\mu} \sim$ $F_{\mu}^{\prime} e^{i \omega_{\mu} t}$, which extend to past times as $F_{\mu} e^{i \omega_{\mu}[t-r / c]}$, along the horizontal dashed lines in Fig. 11a, without shifts. Their propagation is indicated by the line $\psi_{s}$, and is non-expanding.

Chirp bases and spectra were hitherto untreated. Exponential chirp transforms have been used in image processing only to treat non-invariant inputs [29]. All signal applications of chirp transforms have likewise concerned Fourier spectra (cf. [30][32]). It seems that translational asymmetry, or shift variance, made chirp spectra look uninteresting. However, the variance means sensitivity, which would have immense utility [33]. As the shifts result due to how the spectral basis is defined, there is no fundamental dependence on a Doppler rate. We should get such shifts in general by adding a controlled frequency ramp of rate $\beta^{\prime}$ to the DDS of Fig. 2, consistent with a ramped rate in sampling or spectral selection proposed in [33]. Since the receiver axis is one physical point, all such spectra derive from the same waves, as views of the same source physics.

In any case, we finally have a full and precise explanation of the anomaly using no new hypotheses. Our scripts that query JPL Horizons and plot the graphs are posted at https://github. com/earthshrink/flyby-analysis for transparency.

\section{ACKNOWLEDGEMENTS}

This work is owed to followup, persistence and continuing involvement of James K Campbell, who was also first to notice the correlation with transition to SDSTs. The formal notion of wave frequencies varying over time but not space, as in $\psi_{L}$, is owed to Thomas Zaneboni of EPO. Peter G Antreasian kindly provided original graphs for both NEAR and Galileo (Figs. 4, 5 ) and confirmed the radar residuals of Fig. 1 as $5 \sigma$. Comments from other NASA authors have been invaluable in shaping our understanding. Rosetta's flybys could not have been analyzed without the precise tracking times from Trevor Morley of ESA. Lastly, the author thanks colleagues at NAECON for the many comments and discussions.

\section{APPENDIX}

Lemma 1 revisits the necessary and sufficient conditions for satisfying d'Alembert's equation in one dimension. Next, the validity of the exponential chirps $\psi_{E}$ as d'Alembert solutions is established as Theorem 1 . We present a generating theorem 
for expanding wave solutions as the broader result describing the propagation of chirp spectra as in Fig. 11b. The symbol $\omega$ denotes a constant value.

Lemma 1 (d'Alembert conditions). Existence of second order partial derivatives in $t$ and $r$ are necessary and sufficient for any function $\psi(t \mp r / c)$ to satisfy d'Alembert's equation.

Proof. For such a function $\psi(t-r / c)$, writing $\tau=(t-r / c)$, the first and second order partial derivatives

$$
\begin{aligned}
& \psi_{, t} \equiv \frac{\partial \psi}{\partial t} \equiv \frac{d \psi}{d \tau} \frac{\partial \tau}{\partial t}=\frac{d \psi}{d \tau}, \psi_{, t t} \equiv \frac{\partial^{2} \psi}{\partial t^{2}} \equiv \cdots=\frac{d^{2} \psi}{d \tau^{2}}, \\
& \psi_{, r} \equiv \frac{\partial \psi}{\partial r} \equiv \cdots=-\frac{1}{c} \frac{d \psi}{d \tau}, \psi_{, r r} \equiv \frac{\partial^{2} \psi}{\partial r^{2}} \equiv \cdots=\frac{1}{c^{2}} \frac{d^{2} \psi}{d \tau^{2}}
\end{aligned}
$$

follow using the chain rule of differential calculus, and prove that $\psi(\tau)$ satisfies d'Alembert's equation $\psi_{, t t}-c^{2} \psi_{, r r}=0$.

For the complementary function $\psi(t+r / c)$, the first order derivative $\psi_{, r}$ is positive, but the second order derivatives are same. This proves the sufficiency.

The necessity is implicit in the known factorization

$$
\left(\frac{\partial^{2}}{\partial t^{2}}-c^{2} \frac{\partial^{2}}{\partial r^{2}}\right) \psi=\left(\frac{\partial}{\partial t}+c \frac{\partial}{\partial r}\right)\left(\frac{\partial}{\partial t}-c \frac{\partial}{\partial r}\right) \psi=0
$$

that leads to the general solution $f(t+r / c)+g(t-r / c)$.

Theorem 1 (Chirp solutions). d'Alembert's equation in one dimension is satisfied by $\psi(r, t)=\exp \left(i \omega \beta^{-1} e^{\beta[t \mp r / c]}\right)$.

Proof. Writing $\tau=(t \mp r / c)$, so that $\psi=e^{i \omega \beta^{-1}} \exp (\beta \tau)$ and $\partial \tau / \partial r=\mp c^{-1}$, the partial derivatives of $\psi$ are obtained as

$$
\begin{aligned}
\psi_{, r} & \equiv \frac{\partial e^{i \omega \beta^{-1} \exp (\beta \tau)}}{\partial r}=e^{i \omega \beta^{-1} \exp (\beta \tau)} \frac{\partial\left\{i \omega \beta^{-1} \exp (\beta \tau)\right\}}{\partial r} \\
& =\psi \cdot\left(i \omega \beta^{-1}\right) \cdot(\mp / c) \cdot \beta \exp (\beta \tau)=\mp \frac{i \omega}{c} \exp (\beta \tau) \psi
\end{aligned}
$$

and likewise,

$$
\begin{aligned}
\psi_{, t} & \equiv \frac{\partial e^{i \omega \beta^{-1} \exp (\beta \tau)}}{\partial t}=e^{i \omega \beta^{-1} \exp (\beta \tau)} \frac{\partial\left\{i \omega \beta^{-1} \exp (\beta \tau)\right\}}{\partial t} \\
& =\psi \cdot\left(i \omega \beta^{-1}\right) \cdot \beta \exp (\beta \tau)=i \omega \exp (\beta \tau) \psi
\end{aligned}
$$

The second partial derivatives then follow as

$$
\begin{aligned}
\psi_{, r r} & \equiv \frac{\partial}{\partial r}\left[\mp \frac{i \omega}{c} \exp (\beta \tau) \psi\right] \\
& =\frac{\mp i \omega}{c} \frac{\partial \exp (\beta \tau)}{\partial r} \psi \mp \frac{i \omega}{c} \exp (\beta \tau) \cdot \psi_{, r} \\
& =\frac{\mp i \omega}{c} \cdot \beta \exp (\beta \tau) \cdot \frac{\mp 1}{c} \cdot \psi+\frac{i^{2} \omega^{2}}{c^{2}} \exp (2 \beta \tau) \psi \\
& =\frac{i \omega \beta}{c^{2}} \exp (\beta \tau) \psi-\frac{\omega^{2}}{c^{2}} \exp (2 \beta \tau) \psi,
\end{aligned}
$$

and $\quad \psi_{, t t} \equiv \frac{\partial}{\partial t}[i \omega \exp (\beta \tau) \psi]$

$$
\begin{aligned}
& =i \omega \cdot \frac{\partial \exp (\beta \tau)}{\partial t} \psi+i \omega \exp (\beta \tau) \cdot \psi_{, t} \\
& =i \omega \cdot \beta \exp (\beta \tau) \cdot \psi+i^{2} \omega^{2} \exp (2 \beta \tau) \psi \\
& =i \omega \beta \exp (\beta \tau) \psi-\omega^{2} \exp (2 \beta \tau) \psi,
\end{aligned}
$$

which leads to $\left(\partial^{2} / \partial r^{2}-c^{-2} \partial^{2} / \partial t^{2}\right) \psi=0$, as required.

Theorem 2 (Partially invariant functions). The d'Alembert equation is not satisfied by $\psi(r, t)=\exp \left(i \omega e^{\beta t}[t-r / c]\right)$ with frequency varying in time but not over space.

Proof. Its partial derivatives in $r$ would be

$$
\begin{aligned}
\psi_{, r} & \equiv \frac{\partial \psi}{\partial r} \equiv e^{i \omega e^{\beta t} t} \partial e^{-i \omega e^{\beta t} r / c} / \partial r \\
& =e^{i \omega e^{\beta t} t} \cdot\left(-i \omega e^{\beta t} / c\right) e^{-i \omega e^{\beta t} r / c} \\
& =-\frac{i \omega e^{\beta t}}{c} \psi, \\
\text { and } \psi_{, r r} & \equiv-\frac{i \omega}{c} e^{\beta t} \psi_{, r}=-\frac{\omega^{2}}{c^{2}} e^{2 \beta t} \psi .
\end{aligned}
$$

As explained in the Introduction, the partial derivatives with respect to $t$ have additional (highlighted) terms resulting from the asymmetry in $t$ and $r$, as

$$
\begin{aligned}
\psi_{, t} & \equiv \frac{\partial \psi}{\partial t} \equiv \frac{\partial e^{i \omega e^{\beta t}[t-r / c]}}{\partial t} \\
& =e^{i \omega e^{\beta t}[t-r / c]} \cdot i \omega \cdot \frac{\partial e^{\beta t}[t-r / c]}{\partial t} \\
& =\psi \cdot i \omega\left[\beta e^{\beta t}(t-r / c)+e^{\beta t}\right], \\
\text { and } \quad \psi_{, t t} & \equiv \frac{\partial\left(\psi \cdot i \omega\left[\beta e^{\beta t}(t-r / c)+e^{\beta t}\right]\right)}{\partial t} \\
& =\cdots
\end{aligned}
$$

so $\psi_{, t t}$ cannot equal $c^{2} \psi_{, r r}$ except at $t=0$, i.e., at the source. Notice also that $\psi=\psi_{L}$ to first order.

Theorem 3 (Generality of expansion). For each solution $f(t-$ $r / c)$ of d'Alembert's equation $\left(\partial / \partial t^{2}-c^{2} \partial / \partial r^{2}\right) \psi=0$, and every real valued function $g()$ possessing second order partial derivatives in $t$ and $r$, the modified function $h(t, r) \equiv f(g(t-$ $r / c) .[t-r / c])$ is also a solution.

Proof. The modified function retains the argument form $\psi(t-$ $r / c$ ) and would also have second order derivatives in $t$ and $r$, so the result is implied by Lemma 1.

More directly, upon substituting $\tau=(t-r / c)$ as before, we have $\partial \tau / \partial t=1, \partial \tau / \partial r=-c^{-1}, \partial f / \partial \tau \equiv d f / d \tau=f^{\prime}$ and $\partial^{2} f / \partial \tau^{2} \equiv d^{2} f / d \tau^{2}=f^{\prime \prime}$. The first order partial derivatives of the modified function are then

$$
\begin{aligned}
h_{, t} & \equiv \frac{\partial h}{\partial t}=\frac{d f(g \tau)}{d \tau} \frac{\partial \tau}{\partial t}=\frac{d f(g \tau)}{d \tau}=\frac{d f(g \tau)}{d(g \tau)} \frac{d(g \tau)}{d \tau} \\
& =f^{\prime} \cdot\left(\frac{d g}{d \tau} \tau+g \frac{d \tau}{d \tau}\right) \equiv f^{\prime} \cdot\left(g^{\prime} \tau+g\right)
\end{aligned}
$$

using a dot in order to demarcate the parenthesized expression as a factor and not the argument of $f^{\prime}$. We similarly get

$$
h_{, r} \equiv \frac{\partial h}{\partial r}=\frac{d f(g \tau)}{d \tau} \frac{\partial \tau}{\partial r} \equiv-c^{-1} h_{, t} .
$$

The latter proves $h_{, t t}-c^{2} h_{, r r}=0$ is satisfied, since

$$
h_{, r r}=-c^{-1} \frac{h_{, t}}{d \tau} \frac{\partial \tau}{\partial r}=\frac{1}{c^{2}} h_{, t t} .
$$


For completeness, the second order time derivative is

$$
\begin{aligned}
h_{, t t} & \equiv \frac{d\left(f^{\prime} \cdot\left(g^{\prime} \tau+g\right)\right)}{d \tau} \frac{\partial \tau}{\partial t} \\
& =\frac{d f^{\prime}}{d \tau}\left(g^{\prime} \tau+g\right)+f^{\prime} \frac{d\left(g^{\prime} \tau+g\right)}{d \tau} \\
& =f^{\prime \prime} \cdot\left(g^{\prime} \tau+g\right)+f^{\prime}\left(g^{\prime \prime} \tau+g^{\prime}+g\right) .
\end{aligned}
$$

Corollary 3.1 (Exponential chirps). Exponential chirp wave functions, hence constant Doppler rate signals, are expanding.

Proof. The chirp waves of Theorem 1 are obtained by setting $f(\tau)=e^{i \omega \tau}$ and $g(\tau)=e^{\beta \tau}$. Both are d'Alembert solutions by Lemma 1, so the conditions for Theorem 3 are met. Though $g$ can be quite arbitrary, the receiver integration for $F_{\mu}^{\prime}$ (page 6) limits observability to a chirp basis, and thus to monotonic components as shown in Fig. 11b, without loops or oscillations en route to the source.

\section{REFERENCES}

[1] J D Anderson, P A Laing, E L Lau, A S Liu, M Nieto, and S G Turyshev. Study of the anomalous acceleration of Pioneer 10 and 11. Phys Rev D, 65:082004/1-50, Apr 2002. Report LA-UR-00-5654, arXiv:gr-qc/0104064.

[2] J D Anderson, J K Campbell, J E Ekelund, J Ellis, and J F Jordan. Anomalous orbital-energy changes observed during spacecraft flybys of Earth. PRL, 100(9):091102, 2008.

[3] O Bertolami, F Francisco, P J S Gil, and J Páramos. Thermal analysis of the Pioneer Anomaly: A method to estimate radiative momentum transfer. PRD, 78(10):103001, Oct 2008. arXiv:0807.0041v2.

[4] F Francisco, O Bertolami, P J S Gil, and J Páramos. Modelling the reflective thermal contributions to the acceleration of the Pioneer spacecraft. Phys Lett B, 711:337-346, Mar 2011. arXiv:1103.5222.

[5] S G Turyshev, V T Toth, G Kinsella, S C Lee, S M Lok, and J Ellis. Support for the thermal origin of the Pioneer anomaly. Phys Rev Lett, 108, 2012. arXiv: $1204.2507 \mathrm{v} 1$.

[6] G Duff. Kwajalein missile Range: ALTAIR radar contributions to the Space Surveillance Network. Proc Space Control Conf, pages 11-15, 1999.

[7] M L Stone and G P Banner. Radars for the Detection and Tracking of Ballistic Missiles, Satellites, and Planets. Lincoln Lab Jour, 12(2), 2000.

[8] P G Antreasian and J R Guinn. Investigations into the unexpected Delta-V increases during the Earth gravity assists of Galileo and NEAR. AIAA, 1998. 98-4287.

[9] V Guruprasad. Observational evidence for travelling wave modes bearing distance proportional shifts. EPL, 110(5):54001, 2015. arXiv: 1507.08222

[10] J D Giorgini. Status of the JPL Horizons ephemeris system. IAU General Assembly, Aug 2015

[11] P G Antreasian. Pvt comm, 2017.

[12] L Bilbao. Comment on Observational evidence for travelling wave modes bearing distance proportional shifts. EPL, 2016.

[13] L Bilbao. Does the velocity of light depend on the source movement? Prog in Phys, 12:307-312, 2016. arXiv:1606.03921.

[14] S L Adler. Can the flyby anomaly be attributed to earth-bound dark matter? Phys Rev D, 79:023505, Jan 2009. arXiv:0805.2895v4.

[15] S L Adler. Modeling the flyby anomalies with dark matter scattering: update with additional data and further predictions. Int J Mod Phys A, 28, Dec 2011. arXiv:1112.5426.

[16] M D Guman, D C Roth, R Ionasescu, T D Goodson, A H Taylor, and J B Jones. Cassini orbit determination from first Venus flyby to Earth flyby. AAS, 2000. 00-168.

[17] J K Campbell. Pvt comm, 2015-

[18] S Bhaskaran, M Abrahamson, S Chesley, M-K Chung, A Halsell, R Haw, C Helfrich, D Jefferson, B Kennedy, T McElrath, W Owen, B Rush andJ Smith, T-C Wang, and C-W Yen. Navigation of the EPOXI spacecraft to comet Hartley 2. AAS, 2011. 11-486.
[19] P F Thompson, M Abrahamson, S Ardalan, and J Bordi. Reconstruction of the Earth flyby by the Juno spacecraft. AAS, 2014 14-435.

[20] C-C Chen, S Shambayati, A Makovsky, F H Taylor, M I Herman, S H Zingales, C Nuckolls, and K Siemsen. Small Deep Space Transponder (SDST) DS1 Technological Validation Report. Technical report, Descanso NASA, 2000

[21] N R Mysoor, J D Perret, and A W Kermode. Design concepts and performance of NASA X-band (7162 MHx/8415 MHz) transponder for deep-space spacecraft applications. Technical Report 42-104, Descanso NASA, 1991.

[22] R S Bokulic, K E Flaherty, J R Jensen, and T R McKnight. The NEAR spacecraft RF telecommunication system. JHU APL Tech Digest, 19(2), 1998

[23] P F Thompson. Pvt comm, 2019.

[24] C C DeBoy, C B Haskins, T A Brown, R C Schulze, M A Bernacik, J R Jensen, W Millard, D Duven, and S Hill. The RF telecommunications system for the New Horizons mission to Pluto. In IEEEAC, 2003. paper 1369.

[25] C B Haskins, W P Millard, and J R Jensen. Flexible coherent digital transceiver for low power space missions. IEEE Aerospace Conf, 2006.

[26] T Morley and F Budnik. Rosetta navigation at its first earth-swingby. In 19th Intl Symp Space Flight Dynamics, 2006. ISTS 2006-d-52.

[27] T Morley. Pvt comm, 2017.

[28] http://planet4589.org/space/jsr/back/news.447.

[29] G Bonmassar and E L Schwarz. Space-Variant Fourier Analysis: The Exponential Chirp Transform. IEEE Trans. Patt. Anal. and Mach. Int., 19(10):1080-1089, Oct 1997.

[30] P Hartogh and G K Hartmann. High-resolution chirp transform spectrometer for microwave measurements. Meas Sc \& Tech, 1(7):592, 1990.

[31] F Jenet, P Charlton, J Edlund, L Wen, T Creighton, T Prince, and M Tinto. Generalization of the Fast Chirp Transform Algorithm.... Technical report, 2003. http://www.srl.caltech.edu/fct/.

[32] M Mboup and T Adali. A generalization of the Fourier transform and its application to spectral analysis of chirp-like signals. Appl Comput Harmon An, 32:305-312, 2012.

[33] V Guruprasad. A wave effect enabling universal frequency scaling, monostatic passive radar, incoherent aperture synthesis, and general immunity to jamming and interference. In MILCOM (classified session), 2005. arXiv:physics/0812.2652v1. Also: Relaxed bandwidth sharing with Space Division Multiplexing, IEEE WCNC 2005. 